\title{
A Study on Performance Evaluation of Different Parameter Settings of Particle Swarm Optimization in Job Shop Scheduling Problem
}

\author{
Patcharawadee Poolsamran \\ Faculty of Science and Social Sciences \\ Burapha University Sakaeo Campus \\ Sakaeo, THAILAND 27160 \\ patcharp@buu.ac.th
}

\begin{abstract}
This study is to present a particle swarm optimization (PSO) to solve a job shop scheduling problem with the objective of minimizing a makespan. The solution representation of a particle that is encoded with a permutation of all operations on jobs. The operations are ordered by precedence constraints. For the performance evaluation, each particle is evaluated by a quality of the fitness function. In the particle swarm optimization model, the effect of parameters, like the swarm size, on performance are investigated in this paper. In different swarm sizes, the performance of proposed methodology is tested on forty wellknown benchmark problems from the OR-library. The computational results demonstrate that the proposed methodology can effectively solve a job shop scheduling problem and also take less processing time although run on a personal computer.
\end{abstract}

\section{CCS Concepts}

- Computing methodologies $\rightarrow$ Planning and scheduling.

\section{Keywords}

particle swarm optimization (PSO); job shop scheduling problem (JSSP); parameter setting; permutation encoding schema.

\section{INTRODUCTION}

In competitive markets, manufacturer produce their products to compete with specified due dates and production volumes. Therefore, efficient production scheduling has become a critical task of companies and a focusing problem for researchers. In a classification of scheduling problems, there are three basic multimachine problems including an open shop scheduling problem (OSSP), a flow shop scheduling problem (FSSP), and a job shop scheduling problem (JSSP). The current work focuses on the JSSP, in which multiple jobs are processed on multiple machines. Each job must be processed on its own machine order for a predefined processing time. Therefore, a scheduling is to decide when

Permission to make digital or hard copies of all or part of this work for personal or classroom use is granted without fee provided that copies are not made or distributed for profit or commercial advantage and that copies bear this notice and the full citation on the first page. Copyrights for components of this work owned by others than ACM must be honored. Abstracting with credit is permitted. To copy otherwise, or republish, to post on servers or to redistribute to lists, requires prior specific permission and/or a fee. Request permissions from Permissions@acm.org.

ICISS 2019, March 16-19, 2019, Tokyo, Japan

(C) 2019 Association for Computing Machinery.

ACM ISBN 978-1-4503-6103-3/19/03 ..\$15.00

https://doi.org/10.1145/3322645.3322655 process each job on different machines. The challenge here is to determine a feasible sequence of jobs on given machines with an objective of minimizing job completion times.

From past to present, a large number of techniques have been proposed for solving the JSSP, which can be classified into two categories: a mathematical approach and an approximation approach. Historically, the use of mathematic methods, such as integer programming [1] or branch and bound algorithm [2,3], for solving the JSSP were popular because they could achieve an optimal solution. However, the mathematics optimization methods have limitations on the large-scale problems due to polynomialtime solutions. Therefore, a mathematical approach is suitable for small combination problems. That is why many researchers have turned their attention to an approximation approach for solving larger complex problems. In recent years, various methods of an approximation approach have been proposed such as genetic algorithm (GA) [4,5], simulated annealing (SA) [6,7], particle swarm optimization (PSO) [6,7] and so on. It is evident that these powerful algorithms solve the JSSP as well. Although these approximation algorithms have no guarantee achieving optimal solutions, they can offer good solutions within a reasonable runtime.

There are ongoing efforts to improve a performance of the PSO for solving the JSSP, so many variations of the PSO have been proposed. Researchers are interested in studying what influence on finding an optimal solution. Like other approximation methods, a performance of the PSO depends on different factors such as parameter settings, local search method, problem representation, and problem size. In recent years, some studies introduced the PSO-based hybrid models for convergence improvement. For example, Bank el al. [7] compare the performance of the PSO with and without the proposed local search for solving the flow shop scheduling problem. The PSO with local search could have higher efficiency. Perez and Behdinan [8] presented the effect of the different setting parameters and functionality of a particle swarm optimization in classical structural optimization problem. This paper focuses on performance evaluation of different parameter settings of a particle swarm optimization in job shop scheduling problem.

The remainder of the paper is organized as follows: in section 2, the description of the JSSP is given. The standard particle swarm optimization is presented in section 3. Section 4 describes about a methodology and its computational results on test problems. Finally, the conclusions are presented in section 5.

\section{BACKGROUND}




\subsection{Job Shop Scheduling Problem}

The job shop scheduling problems are defined as follows:

(1) A JSSP consists of a set of $n$ jobs $\left(J=\left\{J_{1}, J_{2}, \ldots, J_{i}, \ldots, J_{n}\right\}\right)$ and a set of $m$ machines $\left(M=\left\{M_{1}, M_{2}, \ldots, M_{k}, \ldots, M_{m}\right\}\right)$. Each job is not dependent on each other's job.

(2) Each job $J_{i}$ consists of a predetermined sequence of operations $\left(\mathbf{J}_{\mathrm{i}}=\left\{\mathrm{O}_{\mathrm{i} 1}, \mathrm{O}_{\mathrm{i} 2}, \ldots, \mathrm{O}_{\mathrm{ij}}, \ldots, \mathrm{O}_{\mathrm{ip}}\right\}\right)$; each of which has to move through a set of machines under three constraints. The first is no preemption constraint: when operation has already been started on a specified machine, there is no interruption before it is finished. The second is a resource constraint: each machine can handle only one operation of any job at a time. And the last is a precedence constraint: for maintaining precedence among operations of different jobs, each operation of any job is processed after all predecessor operations had finished.

(3) Each operation of any job is performed on specified machine for a given time.

Figure 1 shows the example of the job shop scheduling problem in $3 \times 3$ size ( 3 jobs $\times 3$ machines $=9$ operations). The first row of example $J_{1}$ has three operations. The first operation, $\mathrm{O}_{11}\left(\mathrm{M}_{1}, 2\right)$, must be operated on the machine $\mathrm{M}_{1}$ in the 2 units of time. After it is finished, the second operation, $\mathrm{O}_{12}\left(\mathrm{M}_{2}, 3\right)$, can be operated on the machine $\mathrm{M}_{2}$ in the 3 units of time. And the last operation is $\mathrm{O}_{13}\left(\mathrm{M}_{3}, 1\right)$ which is operated on the machine $\mathrm{M}_{3}$ in the 1 unit of time. Like the first job, the others are operated on a specified machine within a given time.

\begin{tabular}{|c|c|c|c|}
\hline & Operation 1 & Operation 2 & Operation 3 \\
\hline Job 1 & $\mathrm{O}_{11}\left(\mathrm{M}_{1}, 2\right)$ & $\mathrm{O}_{12}\left(\mathrm{M}_{2}, 3\right)$ & $\mathrm{O}_{13}\left(\mathrm{M}_{3}, 1\right)$ \\
\hline Job 2 & $\mathrm{O}_{21}\left(\mathrm{M}_{2}, 3\right)$ & $\mathrm{O}_{22}\left(\mathrm{M}_{1}, 1\right)$ & $\mathrm{O}_{23}\left(\mathrm{M}_{3}, 2\right)$ \\
\hline Job 3 & $\mathrm{O}_{31}\left(\mathrm{M}_{2}, 2\right)$ & $\mathrm{O}_{32}\left(\mathrm{M}_{3}, 3\right)$ & $\mathrm{O}_{33}\left(\mathrm{M}_{1}, 2\right)$ \\
\hline & $\begin{array}{l}\mathrm{ij}\left(\mathrm{M}_{\mathrm{k}}, \mathrm{T}\right) \\
\begin{aligned} \mathrm{O}_{\mathrm{ij}} & \text { an ope } \\
\mathrm{M}_{\mathrm{k}} & \text { a mac } \\
\mathrm{T} & \text { a proc }\end{aligned}\end{array}$ & $\mathrm{k}$ & a machine $\mathrm{k}$ \\
\hline
\end{tabular}

Figure 1. Example of a 3x3 JSSP.

From Figure 1, the example of 3x3 JSSP can be represented using a disjunctive graph as shown in Figure 2. The operations which belong to the same job are connected by conjunctive arcs. While the operations that must be performed in the same machine are connected by disjunctive arcs.
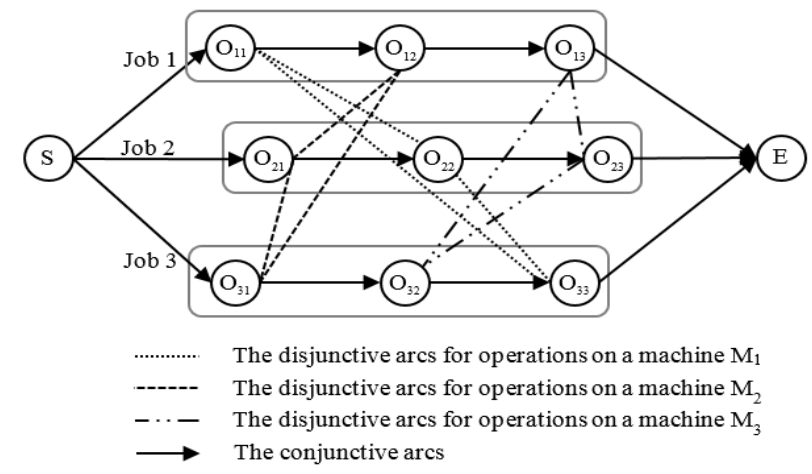

Figure 2. A disjunctive graph of 3x3 JSSP.

\subsection{Particle Swarm Optimization}

Particle swarm optimization was first introduced by Kennedy and Eberhart [10,11] in 1995. The PSO is a population-based optimization algorithm inspired by social behavior of bird flocking or fish schooling. Some birds or fish live as social groups in order to look for foods, migrate, and be protected from predators to survive. In foraging behavior, each individual communicates with others to share their own experience and use the experience of the swarm leader in order to find foods in the shortest possible time. These are used for guiding optimization search by simulating a swarm of particle fly to find foods in the solution space.

In the standard PSO, there is a swarm consisting of $\mathrm{N}$ particles where each particle $i$ has a position vector, $\vec{X}_{i}$ and a velocity vector, $\vec{V}_{i}$ in D-dimensional size as equation (1) and (2), respectively.

$$
\begin{aligned}
& \vec{X}_{i}(t)=\left\{x_{1}(t), x_{2}(t), \ldots, x_{D}(t)\right\} \\
& \vec{V}_{i}(t)=\left\{v_{1}(t), v_{2}(t), \ldots, v_{D}(t)\right\}
\end{aligned}
$$

In every iteration, the two best values, pBest and gBest, are updated by each particle. Individual particles keep their own personal best, pBest, which are associated with the best position that has archived so far. The global best, gBest, is the best position found among all particles in the swarm. Once pBest and gBest are updated, each particle must adjust its velocity vector according to equation (3), (4) and (5).

$$
\begin{aligned}
& \vec{V}_{i}(t+1)=w \times \vec{V}_{i}(t)+p_{i}(t)+g_{i}(t) \\
& p_{i}(t)=c_{1} \times \text { rand } \times\left(\text { pBest }-\vec{X}_{i}(t)\right) \\
& g_{i}(t)=c_{2} \times \text { rand } \times\left(\text { gBest }-\vec{X}_{i}(t)\right)
\end{aligned}
$$

where $\vec{V}_{i}(t+1)$ is a new velocity vector for next iteration, $w$ is the inertia weight, the constant $c_{1}$ and $c_{2}$ are the cognitive parameter in itself and the social parameter in the swarm, and rand is a random number.

$$
\vec{X}_{i}(t+1)=\vec{X}_{i}(t)+\vec{V}_{i}(t+1)
$$

After the new velocity vectors are calculated, each particle can move to its new position vector by using the equation (6). The searching needs to be repeated until some stopping criteria is met, for example, the maximum number of iteration, and the minimum error.

\section{PROPOSED METHODOLOGY}

There are three main steps for a study on performance evaluation of different parameter setting of particle swarm optimization for solving the JSSP, which are as follows:

\subsection{Problem representation}

For solving a job shop scheduling problem by the PSO algorithm, the first step is to choose a type of solution encoding. This study is to represent a position of each particle with a real encoding and then transform it into a permutation encoding. From an example of $3 \times 3$ JSSP in Figure 1, the position of the particle is formed as a problem solution by a vector shown in Figure 3(a). A particle vector is created with a length of $3 \times 3$ dimensions and with each dimension set to a value of random real values in the range of 0 to 
1. After that a real-valued particle is transformed into a vector of integer from 1 to 9 according to its ordering. As shown in Figure $3(b)$, the integer vector is denoted by a job series. Finally, the position of particle formed as a job series is replaced by its 9 operations in given precedence of each job.

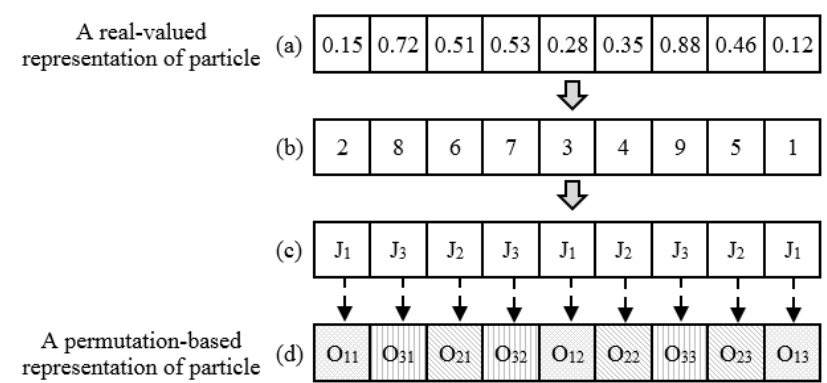

Figure 3. A representation of a particle for a $3 \times 3$ JSSP.

\subsection{Fitness evaluation}

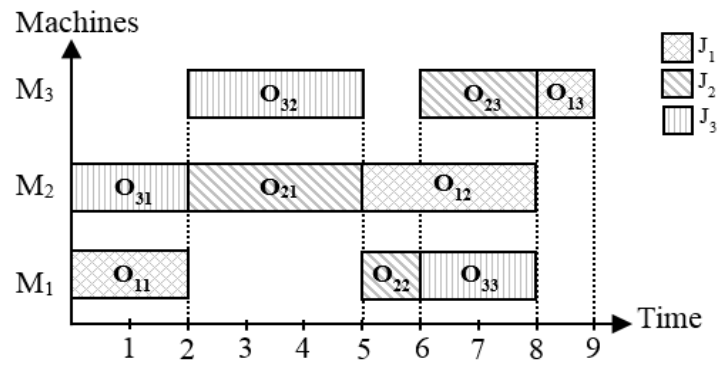

Figure 4. A Gantt chart representation for a $3 \times 3$ solution.

The fitness function is used to evaluate the performance of each particle. For adapting the PSO to the JSSP, the fitness function is mapped to the objective function, that is to minimize the makespan value. Therefore, the solution represented by the positon of the particle is used to calculate a makespan value by assignment operations on a given machine under problem constraints. From the example of permutation-based particle in Figure 3(d), That can be calculated the maximum of completetime on all machines (called makespan) for 9 units of time as shown in Figure 4.

\subsection{PSO solution for the JSSP}

The process for implementing the PSO solution for the JSSP is described as follows:

Step 1: Initialize the parameter setting.

Step 2: Generate a swarm of particles, which is represented with a set of position vectors. A particle position is initialized with a random number in the range of $[0,2]$.

Step 3: Generate a particle velocity of each member of a swarm with a random number in the range of $[-2,2]$.

Step 4: Evaluate the performance of each member of a swarm by calculating a makespan through decoding its own position.

Step 5: Find the local best position (called pBest) by comparison the current fitness value with the previous value. If the current fitness value is better than, then set it to the fitness value of pBest and also set the current position to the position of pBest.

Step 6: Find the global best particle (called gBest) by comparison the current fitness value with the best fitness value of swam. Like the local best particle, if the current fitness value is better than, then set it to the fitness value of gBest and also set the current position to the position of gBest.

Step 7: Update the position and the velocity of each particle in a swarm.

Step 8: Repeat step 2-7 until a maximum number of iterations is reached.

From above mentioned, the PSO model is illustrated as a flowchart in Figure 5.

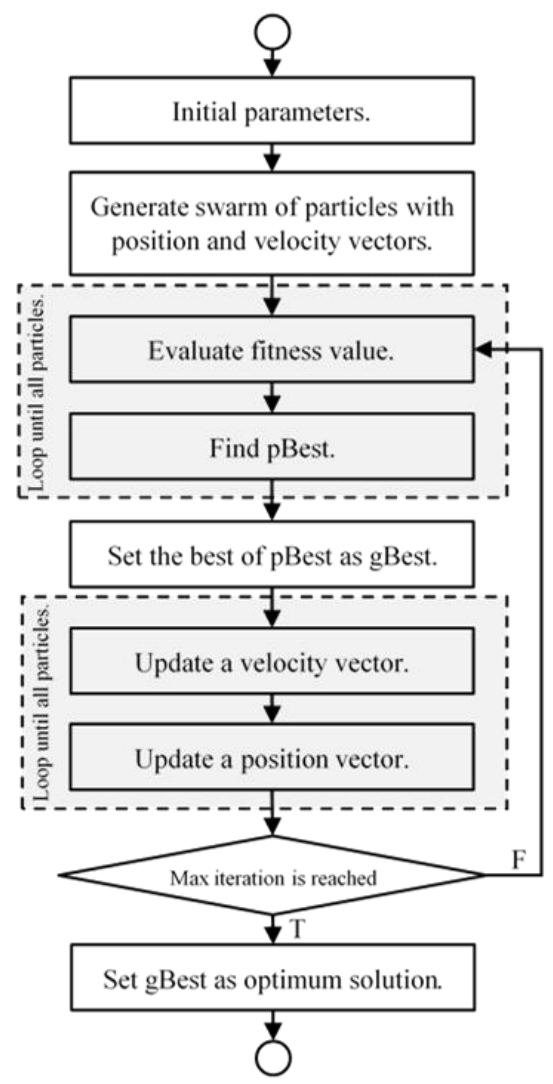

Figure 5. Flowchart of the PSO for the JSSP.

\section{COMPUTATIONAL RESULTS}

The methodology of particle swarm optimization was implemented in JAVA and ran on Intel ${ }^{\circledR}$ Core $^{\mathrm{TM}} 2$ Duo CPU E7500 $2.93 \mathrm{GHz}$. with $4 \mathrm{~GB}$ RAM. The presented model was tested on 40 instances from the OR-library [9]. For the purpose of study on the parameter settings, the parameter values of PSO are set as follows: the constant $\mathrm{C}_{1}$ and $\mathrm{C}_{2}$ are set to 2 , the inertia weight $w$ is set to 1 , the maximum number of generations is set to 1,000 , and swarm size of initial population is set to $20,40,60$, and 80. For each selected swarm size, each instance is randomly performed 10 times. 
Table 1. Computational results of $\mathbf{4 0}$ difference instances

\begin{tabular}{|c|c|c|c|c|c|c|c|c|c|c|c|c|c|c|}
\hline \multirow{2}{*}{ Instance } & \multicolumn{2}{|c|}{ Size } & \multicolumn{3}{|c|}{20} & \multicolumn{3}{|c|}{40} & \multicolumn{3}{|c|}{60} & \multicolumn{3}{|c|}{80} \\
\hline & $\mathrm{m}$ & $\mathrm{n}$ & $\mathrm{BS}$ & $\mathrm{RE}$ & CT & $\mathrm{BS}$ & $\mathrm{RE}$ & $\mathrm{CT}$ & $\mathrm{BS}$ & $\mathrm{RE}$ & CT & $\mathrm{BS}$ & $\mathrm{RE}$ & CT \\
\hline la02 & 10 & 5 & 815 & 0.24 & $00: 15$ & 655 & 0.00 & $00: 29$ & 655 & 0.00 & $00: 46$ & 655 & 0.00 & 01:00 \\
\hline $\mathrm{la} 03$ & 10 & 5 & 755 & 0.26 & $00: 15$ & 597 & 0.00 & $00: 30$ & 597 & 0.00 & $00: 46$ & 597 & 0.00 & 01:01 \\
\hline la04 & 10 & 5 & 745 & 0.26 & $00: 15$ & 734 & 0.24 & 00:30 & 691 & 0.17 & $00: 45$ & 705 & 0.19 & 01:01 \\
\hline la05 & 10 & 5 & 593 & 0.00 & $00: 15$ & 613 & 0.03 & $00: 29$ & 616 & 0.04 & $00: 45$ & 612 & 0.03 & 01:00 \\
\hline la06 & 15 & 5 & 926 & 0.00 & $00: 26$ & 926 & 0.00 & 00:51 & 926 & 0.00 & $01: 16$ & 926 & 0.00 & 01:42 \\
\hline la07 & 15 & 5 & 1074 & 0.21 & $00: 25$ & 1098 & 0.23 & $00: 51$ & 1070 & 0.20 & $01: 17$ & 1050 & 0.18 & 01:42 \\
\hline la08 & 15 & 5 & 1087 & 0.26 & $00: 26$ & 1053 & 0.22 & $00: 51$ & 1042 & 0.21 & 01:17 & 1010 & 0.17 & 01:43 \\
\hline la09 & 15 & 5 & 1110 & 0.17 & $00: 25$ & 1061 & 0.12 & 00:49 & 1141 & 0.20 & $01: 17$ & 1100 & 0.16 & $01: 42$ \\
\hline la10 & 15 & 5 & 1044 & 0.09 & $00: 25$ & 1053 & 0.10 & 00:50 & 1020 & 0.06 & $01: 16$ & 1004 & 0.05 & 01:43 \\
\hline la11 & 20 & 5 & 1487 & 0.22 & $00: 37$ & 1390 & 0.14 & $01: 16$ & 1399 & 0.14 & 01:53 & 1382 & 0.13 & $02: 32$ \\
\hline la12 & 20 & 5 & 1228 & 0.18 & 00:39 & 1176 & 0.13 & 01:17 & 1172 & 0.13 & 01:54 & 1186 & 0.14 & $02: 33$ \\
\hline la13 & 20 & 5 & 1326 & 0.15 & 00:38 & 1370 & 0.19 & $01: 16$ & 1303 & 0.13 & 01:53 & 1297 & 0.13 & $02: 34$ \\
\hline la14 & 20 & 5 & 1373 & 0.06 & 00:38 & 1387 & 0.07 & $01: 16$ & 1397 & 0.08 & $01: 53$ & 1355 & 0.05 & $02: 32$ \\
\hline la15 & 20 & 5 & 1580 & 0.31 & 00:38 & 1470 & 0.22 & $01: 16$ & 1549 & 0.28 & 01:56 & 1486 & 0.23 & $02: 32$ \\
\hline la16 & 10 & 10 & 1232 & 0.30 & 00:33 & 1220 & 0.29 & 01:06 & 1250 & 0.32 & 01:39 & 1158 & 0.23 & $02: 12$ \\
\hline la17 & 10 & 10 & 1011 & 0.29 & 00:33 & 1068 & 0.36 & 01:07 & 1005 & 0.28 & 01:39 & 1075 & 0.37 & $02: 14$ \\
\hline la18 & 10 & 10 & 1191 & 0.40 & $00: 33$ & 1159 & 0.37 & 01:06 & 1088 & 0.28 & 01:39 & 1125 & 0.33 & $02: 12$ \\
\hline la19 & 10 & 10 & 1184 & 0.41 & $00: 33$ & 1210 & 0.44 & 01:06 & 1162 & 0.38 & 01:38 & 1116 & 0.33 & $02: 13$ \\
\hline la20 & 10 & 10 & 1180 & 0.31 & $00: 33$ & 1230 & 0.36 & 01:06 & 1134 & 0.26 & 01:39 & 1212 & 0.34 & 02:11 \\
\hline la21 & 15 & 10 & 1688 & 0.61 & $00: 57$ & 1561 & 0.49 & $01: 54$ & 1528 & 0.46 & $02: 53$ & 1577 & 0.51 & 03:53 \\
\hline la22 & 15 & 10 & 1497 & 0.61 & $00: 58$ & 1419 & 0.53 & $01: 53$ & 1447 & 0.56 & $02: 56$ & 1433 & 0.55 & $03: 56$ \\
\hline la23 & 15 & 10 & 1493 & 0.45 & 00:58 & 1516 & 0.47 & $01: 54$ & 1356 & 0.31 & $02: 53$ & 1544 & 0.50 & 03:52 \\
\hline la24 & 15 & 10 & 1464 & 0.57 & 00:57 & 1340 & 0.43 & 01:56 & 1424 & 0.52 & $02: 52$ & 1321 & 0.41 & 03:52 \\
\hline la25 & 15 & 10 & 1474 & 0.51 & 00:58 & 1399 & 0.43 & $01: 56$ & 1457 & 0.49 & $02: 54$ & 1489 & 0.52 & $03: 53$ \\
\hline la26 & 20 & 10 & 1875 & 0.54 & $01: 26$ & 1897 & 0.56 & $02: 55$ & 1861 & 0.53 & $04: 23$ & 1902 & 0.56 & 05:53 \\
\hline la27 & 20 & 10 & 2023 & 0.64 & $01: 25$ & 1993 & 0.61 & $02: 51$ & 2065 & 0.67 & $04: 21$ & 1974 & 0.60 & 05:49 \\
\hline la28 & 20 & 10 & 1936 & 0.59 & $01: 25$ & 1841 & 0.51 & $02: 50$ & 1865 & 0.53 & $04: 18$ & 1849 & 0.52 & $05: 51$ \\
\hline la29 & 20 & 10 & 1899 & 0.65 & $01: 27$ & 1839 & 0.60 & $02: 51$ & 1839 & 0.60 & $04: 21$ & 1851 & 0.61 & $05: 51$ \\
\hline la30 & 20 & 10 & 2007 & 0.48 & $01: 24$ & 1878 & 0.39 & $02: 50$ & 1975 & 0.46 & $04: 19$ & 1872 & 0.38 & 05:49 \\
\hline la31 & 30 & 10 & 2611 & 0.46 & $02: 37$ & 2483 & 0.39 & $05: 23$ & 2381 & 0.33 & 08:05 & 2512 & 0.41 & $10: 57$ \\
\hline la32 & 30 & 10 & 2733 & 0.48 & $02: 36$ & 2735 & 0.48 & 05:31 & 2722 & 0.47 & 08:18 & 2620 & 0.42 & $10: 52$ \\
\hline la33 & 30 & 10 & 2520 & 0.47 & $02: 36$ & 2386 & 0.39 & $05: 17$ & 2432 & 0.41 & 08:04 & 2438 & 0.42 & $10: 57$ \\
\hline la34 & 30 & 10 & 2502 & 0.45 & $02: 36$ & 2482 & 0.44 & $05: 18$ & 2464 & 0.43 & 08:03 & 2498 & 0.45 & $11: 01$ \\
\hline la35 & 30 & 10 & 2780 & 0.47 & $02: 55$ & 2782 & 0.47 & $05: 20$ & 2695 & 0.43 & 08:08 & 2677 & 0.42 & $10: 57$ \\
\hline la36 & 15 & 15 & 1919 & 0.51 & 01:31 & 1880 & 0.48 & 03:05 & 1889 & 0.49 & $04: 41$ & 1896 & 0.50 & $06: 19$ \\
\hline la37 & 15 & 15 & 2176 & 0.56 & 01:32 & 2163 & 0.55 & 03:06 & 2077 & 0.49 & $04: 47$ & 2077 & 0.49 & $06: 26$ \\
\hline la38 & 15 & 15 & 1902 & 0.59 & 01:34 & 1889 & 0.58 & 03:09 & 1868 & 0.56 & $04: 43$ & 1847 & 0.54 & 06:19 \\
\hline la39 & 15 & 15 & 1927 & 0.56 & 01:32 & 1870 & 0.52 & 03:05 & 1925 & 0.56 & $04: 42$ & 1904 & 0.54 & $06: 21$ \\
\hline la40 & 15 & 15 & 2058 & 0.68 & 01:33 & 1984 & 0.62 & 03:06 & 1906 & 0.56 & $04: 40$ & 1957 & 0.60 & $06: 20$ \\
\hline
\end{tabular}

Table 1 shows the computational results of different instances. The best solution column (BS) denotes the minimum makespan, the relative percent error column (RE) denotes a percentage of best solution over best known solution, and the computation time column (CT) denotes an average CPU time.

\section{CONCLUSION}

The computational experiments show the efficiency of the algorithm in finding an optimum solution for difference size problems. Moreover, the particle swarm optimization is easy to implement and can be applied in the small and medium-sized enterprises which computer resources are limited. Future research will modify the standard PSO and then address more challenging optimization problems in the real world.

\section{ACKNOWLEDGMENTS}

The author would like to thank Faculty of Science and Social Sciences, Burapha University Sakaeo Campus for financial support.

\section{REFERENCES}

[1] Ku, W.Y., and Beck, J.C. 2016. Mixed Integer Programming models for job shop scheduling: A computational analysis. 
Computers \& Operations Research. 73 (September 2016), 165-173. DOI=https://doi.org/10.1016/j.cor.2016.04.006.

[2] AitZai, A., Benmedjdoub, B. and Boudhar, M. 2016. Branchand-bound and PSO algorithms for no-wait job shop scheduling. J Intell Manuf. 27, 3 (June 2016), 679-688. DOI=https://doi.org/10.1007/s10845-014-0906-7.

[3] Brucker, P., Burke, E.K. and Groenemeyer, S. 2012. A branch and bound algorithm for the cyclic job-shop problem with transportation. Computers \& Operations Research. 39, 12 (December 2012), 3200-3214. DOI=https://doi.org/10.1016/j.cor.2012.04.008.

[4] Bhatt, N. and Chauhan, N. R. 2015. Genetic algorithm applications on Job Shop Scheduling Problem: A review. In Proceeding of the 2015 International Conference on Soft Computing Techniques and Implementations, (Faridabad, India, October 8-10, 2015). ICSCTI'15. IEEE Computer Society, 7-14. DOI=http://doi.org/10.1109/ICSCTI.2015.7489556.

[5] Kundakc1, N. and Kulak, O. 2016. Hybrid genetic algorithms for minimizing makespan in dynamic job shop scheduling problem. Computers \& Industrial Engineering. 96 (June 2016), 31-51. DOI=https://doi.org/10.1016/j.cie.2016.03.011.

[6] Zhang, X.F., Koshimura, M., Fujita, H., and Hasegawa, R. 2011. Combining PSO and local search to solve scheduling problems. In Proceedings of the 13th annual conference companion on Genetic and evolutionary computation, (Dublin, Ireland, July 12 - 16, 2011). GECCO'11. ACM,
New York, NY, 347-354. DOI= https://doi.org/10.1145/2001858.2002017.

[7] Bank, M., Fatemi Ghomi, S.M.T., Jolai, F. and Behnamian, J. 2012. Application of particle swarm optimization and simulated annealing algorithms in flow shop scheduling problem under linear deterioration. Advances in Engineering Software. 47, 1 (May 2012), 1-6. DOI= https://doi.org/10.1016/j.advengsoft.2011.12.001.

[8] Perez, R. E. and Behdinan, K. 2007. Particle swarm approach for structural design optimization. Computers and Structures, $85,1579-1588$. DOI= https://doi.org/10.1016/j.compstruc.2006.10.013.

[9] Beasley, J.E. 1990. OR-Library: Distributing Test Problems by Electronic Mail. Journal of the Operations Research Society. 41, 11, 1069-1072. DOI= https://doi.org/10.2307/2582903

[10] Kennedy, J. and Eberhart, R. 1995. Particle swarm optimization. In Proceedings of IEEE international conference on neural network, (Perth, Australia, 27 November - 1 December, 1995). ICNN'95. 1942-1948. DOI= http://dx.doi.org/10.1109/ICNN.1995.488968.

[11] Eberhart, R. and Kennedy, J. 1995. A new optimizer using particle swarm theory. In Proceedings of Sixth International Symposium on Micro Machine and Human Science, (Nagoya, Japan, October 4 - 6, 1995). MHS'95. 39-43. DOI= http://dx.doi.org/10.1109/MHS.1995.494215. 